\title{
Add-on LABA in a separate inhaler as asthma step-up therapy versus increased dose of ICS or ICS/LABA combination inhaler
}

David B. Price ${ }^{1,2}$, Gene Colice ${ }^{3}$, Elliot Israel ${ }^{4}$, Nicolas Roche ${ }^{5}$, Dirkje S. Postma ${ }^{6}$, Theresa W. Guilbert" ${ }^{7}$, Willem M.C. van Aalderen ${ }^{8}$, Jonathan Grigg ${ }^{9}$, Elizabeth V. Hillyer ${ }^{2}$, Victoria Thomas ${ }^{2}$ and Richard J. Martin ${ }^{10}$

Affiliations: ${ }^{1}$ Academic Primary Care, University of Aberdeen, Aberdeen, UK. ${ }^{2}$ Observational and Pragmatic Research Institute, Singapore, Singapore. ${ }^{3}$ Washington Hospital Center and George Washington University School of Medicine, Washington, DC, USA. "Brigham and Women's Hospital, Harvard Medical School, Boston, MA, USA. ${ }^{5}$ University Paris Descartes (EA2511), Cochin Hospital Group (AP-HP), Paris, France. ${ }^{6}$ University of Groningen, University Medical Center Groningen, Groningen, The Netherlands. ${ }^{7}$ Cincinnati Children's Hospital and Medical Center, Cincinnati, OH, USA. ${ }^{8}$ Emma Children's Hospital AMC, Amsterdam, The Netherlands. ${ }^{9}$ Blizard Institute, Queen Mary University of London, London, UK. ${ }^{10}$ National Jewish Health and the University of Colorado Denver, Denver, CO, USA.

Correspondence: David Price, Academic Primary Care, Division of Applied Health Sciences, University of Aberdeen, Polwarth Building, Foresterhill, Aberdeen, AB25 2ZD, UK. E-mail: dpricearirl.org

ABSTRACT Asthma management guidelines recommend adding a long-acting $\beta_{2}$-agonist (LABA) or increasing the dose of inhaled corticosteroid (ICS) as step-up therapy for patients with uncontrolled asthma on ICS monotherapy. However, it is uncertain which option works best, which ICS particle size is most effective, and whether LABA should be administered by separate or combination inhalers.

This historical, matched cohort study compared asthma-related outcomes for patients (aged 12-80 years) prescribed step-up therapy as a $\geqslant 50 \%$ extrafine ICS dose increase or add-on LABA, via either a separate inhaler or a fine-particle ICS/LABA fixed-dose combination (FDC) inhaler. Risk-domain asthma control was the primary end-point in comparisons of cohorts matched for asthma severity and control during the baseline year.

After 1:2 cohort matching, the increased extrafine ICS versus separate ICS+LABA cohorts included 3232 and 6464 patients, respectively, and the fine-particle ICS/LABA FDC versus separate ICS+LABA cohorts included 7529 and 15058 patients, respectively (overall mean age 42 years; 61-62\% females). Over one outcome year, adjusted OR (95\% CI) for achieving asthma control were 1.25 (1.13-1.38) for increased ICS versus separate ICS+LABA and 1.06 (1.05-1.09) for ICS/LABA FDC versus separate ICS+LABA.

For patients with asthma, increased dose of extrafine-particle ICS, or add-on LABA via ICS/LABA combination inhaler, is associated with significantly better outcomes than ICS+LABA via separate inhalers.

@ERSpublications

Increased extrafine ICS or fixed-dose combination ICS/LABA is more effective than ICS and LABA in separate inhalers http://ow.ly/ZtNYe

This article has supplementary material available from openres.ersjournals.com

Received: Dec 312015 | Accepted after revision: March 092016

Clinical trial: This study is registered at clinicaltrials.gov with identifier NCT01697722

Support statement: The analyses were funded by Teva Pharmaceuticals Limited (Petach Tikva, Israel). Access to data from the Optimum Patient Care Research Database was co-funded by Research in Real-Life Ltd (Cambridge, UK). Funding information for this article has been deposited with Open Funder Registry

Conflict of interest: Disclosures can be found alongside this article at openres.ersjournals.com

The content of this work is @ the authors or their employers. Design and branding are @ERS 2016. This article is open access and distributed under the terms of the Creative Commons Attribution Non-Commercial Licence 4.0. 


\section{Introduction}

For patients whose asthma is not controlled with low-to-moderate doses of inhaled corticosteroids (ICS), the preferred initial step-up option in asthma management guidelines is the addition of a long-acting $\beta_{2}$-agonist (LABA), either as a fixed-dose combination (FDC) or via two separate inhalers, with further step-up options including an increased dose of ICS [1, 2]. Until 2011, the British Thoracic Society/Scottish Intercollegiate Network recommended ICS and LABA in either separate or combination inhalers [3]. However, previous research has shown that, for patients using separate ICS and LABA inhalers, problems with adherence can result in LABA monotherapy, which has been associated with an increased risk of death [4]. Despite this, there is little evidence or guidance in the literature regarding whether separate or combination inhalers are preferred or recommended.

For adolescents and adults, the addition of LABA to ICS therapy often provides better symptom control and less need for reliever therapy [5-7]. In randomised controlled trials, patients receiving LABA in combination with ICS experienced improved lung function and symptom control compared with patients receiving an increased ICS dose [6,8-13]. Some of these trials assessed the effect of combination inhalers compared with separate inhalers, but few statistically significant differences were observed [12]. However, up to $95 \%$ of patients with asthma are not eligible for participation in randomised controlled trials because of smoking, comorbidities or lung function impairment [14]. Furthermore, recruited patients benefit from close follow-up during their participation in these trials, and adherence in these studies is therefore likely to be higher than in the general patient population $[15,16]$. Observational studies have indeed suggested that combination inhalers improve adherence in real-life patients, which is likely to influence therapeutic outcomes [17, 18]. Moreover, we have previously shown that an increased dose of ICS is as effective as add-on LABA via FDC inhaler in a general population of patients with asthma [19].

Another variable that may affect outcomes is particle size. Previous randomised trials have often focused on fine-particle ICS (mass median aerodynamic diameter $2-5 \mu \mathrm{m}$ ), such as fluticasone and budesonide, whereas a substantial proportion of the general asthma patient population is prescribed extrafine-particle ICS. Extrafine-particle ICS (e.g. beclomethasone, mass median aerodynamic diameter $\sim 1 \mu \mathrm{m}$ ), exhibits significantly increased lung deposition compared with fine-particle ICS [20, 21]. Furthermore, real-life research has suggested that treatment with extrafine-particle ICS results in similar or superior asthma-related outcomes at lower prescribed doses compared with fine-particle ICS [22-25].

The objective of this retrospective matched cohort study was to evaluate the real-life effectiveness of step-up asthma therapy with LABA added to ICS (any particle size) via separate inhalers compared with either an increased dose of extrafine-particle ICS or with a fine-particle ICS/LABA FDC inhaler. Our hypothesis was that, for adolescent and adult patients with evidence of persistent uncontrolled asthma, step-up treatments with either increased extrafine-particle ICS or with fine-particle ICS/LABA FDC would be a superior alternative to ICS and LABA in separate inhalers.

\section{Methods}

Data source and patients

This historical, matched cohort, comparative effectiveness study drew on two large UK databases: the General Practice Research Database (GPRD), which is now part of the Clinical Practice Research Datalink [26], and the Optimum Patient Care Research Database (OPCRD) (supplementary material) [27]. Both databases are well described and have previously been used for respiratory research. The Independent Scientific Advisory Committee approved the use of GPRD data for the purpose of this study, and the OPCRD was approved by Trent Multi-Centre Research Ethics Committee (current NHS HRA approval REC reference 15/EM/0150) for clinical research use. The study protocol was designed by an independent steering committee and approved by the Anonymised Data Ethics Protocols and Transparency Committee, which is the independent scientific advisory committee of Optimum Patient Care. This study was registered at clinicaltrials.gov (identifier NCT01697722).

Eligible patients (aged 12-80 years) had at least 2 years of continuous data within the study window: a 1-year baseline period before the index date to control for baseline confounders, and a 1-year outcome period following the index date during which primary and secondary outcome parameters were assessed. During the baseline year, patients had at least two prescriptions for asthma therapy, including at least one for ICS. Exclusion criteria included a recorded diagnosis of chronic obstructive pulmonary disease (COPD) or any chronic respiratory disease other than asthma, as well as a history of smoking for patients between 61 and 80 years of age. This age limit was chosen in order to minimise the inclusion of patients with COPD misdiagnosed as asthma, since the proportion of patients with airflow obstruction who also have COPD increases to $\sim 40 \%$ for patients aged 60-69 years and the diagnoses can be difficult to differentiate [28-30].

The index date was defined as the date ICS treatment was stepped up by one of the following three options: 1) $\geqslant 50 \%$ increase in ICS dose of extrafine-particle beclomethasone dipropionate (ICS step-up); 
2) addition of LABA via FDC of fine-particle ICS and LABA with no change in ICS dose (ICS/LABA FDC); or 3) addition of LABA by separate inhaler, with no change in ICS drug substance, device type or daily ICS dose in beclomethasone equivalents (separate ICS+LABA). During the baseline period, patients in all cohorts were prescribed either fine-particle or extrafine-particle ICS. During the outcome period, patients in the ICS step-up cohort were prescribed extrafine-particle ICS, patients in the ICS/LABA FDC cohort received fine-particle ICS, and patients in the separate ICS+LABA cohort received either fine-particle or extrafine-particle ICS (supplementary material and table S2). The study period covered 14 years between January 1997 and January 2011.

\section{Outcome measures}

The primary effectiveness end-point was risk-domain asthma control, i.e. no hospital admission, no emergency department attendance, no general practice attendance for lower respiratory tract infections, and no oral corticosteroid prescriptions as previously described [23, 25]. Secondary end-points included the number of severe exacerbations, number of acute respiratory events and treatment stability (table 1) [31, 32].

The total daily coverage of $\beta_{2}$-agonist (short-acting $\beta_{2}$-agonist $(\mathrm{SABA})+\mathrm{LABA}$ ) was calculated as the dispensed amount divided by 365 and defining two puffs of SABA (albuterol $200 \mu \mathrm{g}$ or terbutaline $500 \mu \mathrm{g}$ ) as lasting $4 \mathrm{~h}$, and two puffs of LABA via pressurised metered-dose inhaler or one puff of LABA via dry powder inhaler as lasting $12 \mathrm{~h}$.

\section{Statistical analysis}

The statistical analysis plan was defined before data analysis, following a priori defined standard procedures of the research team. After patient selection (figure S1), an exploratory analysis was carried out for all

\section{TABLE 1 Study definitions: database-derived outcome measures and matching criteria}

\section{Primary end-point}

Risk-domain asthma control includes all of the following:

1. No asthma-related ${ }^{\#}$ hospital attendance or admission, ED attendance, out-of-hours attendance, or outpatient hospital attendance, and

2. No GP consultation for lower respiratory tract infection" with resultant antibiotic prescription", and

3. No prescription for acute course of oral corticosteroids

\section{Secondary end-points}

Number of severe exacerbations ${ }^{+}$, defined as any of the following [31]:

1. Asthma-related ${ }^{\#}$ hospital attendance or admission or ED attendance, or

2. Acute course of oral corticosteroids

Number of acute respiratory events, defined as any of the following:

1. Asthma-related ${ }^{\#}$ hospital attendance or admission or ED attendance, or

2. Acute course of oral corticosteroids, or

3. GP consultation for lower respiratory tract infection"

Treatment stability, includes all of the following:

1. Asthma control (primary end-point, see above) and

2. No treatment change, defined additional therapy or change in therapy as

a) Increased ICS dose (by $\geqslant 50 \%$ ), or

b) Use of additional therapy, such as LABA, LTRA or theophylline

Matching criteria: patients were matched at the index prescription date by

1. Sex (male/female)

2. Age ( \pm 5 years)

3. Last ICS daily dose prescribed before index date prescription (categorised as $1-50 \mu \mathrm{g} / 51-100 \mu \mathrm{g} /$ $101-200 \mu \mathrm{g} / 201-300 \mu \mathrm{g} / 301-400 \mu \mathrm{g} />400 \mu \mathrm{g}$ in extrafine beclomethasone equivalents ${ }^{\S}$ ]

4. Asthma control (defined as primary end-point) during baseline year (controlled/not controlled)

5. Mean SABA daily dose during baseline year ${ }^{f}$ (categorised as $0 \mu \mathrm{g} / 1-200 \mu \mathrm{g} / 201-400 \mu \mathrm{g} />400 \mu \mathrm{g}$ )

6. Asthma consultation(s) without severe exacerbation $(0 / 1 / \geqslant 2)$

ED: emergency department; GP: general practitioner; ICS: inhaled corticosteroid; LABA: long-acting $\beta_{2}$-agonist; LTRA: leukotriene receptor antagonist; SABA: short-acting $\beta_{2}$-agonist. ${ }^{\#}$ : defined as all events with a lower respiratory code, including all asthma codes and lower respiratory tract infection codes; ${ }^{\text {I: }}$ identified as GP consultations with a code for lower respiratory tract infection Read code in the database; ${ }^{+}$: hospital attendance/admission and/or an oral corticosteroid course within a 2-week window were considered as one exacerbation; §: doses of budesonide and the large-particle beclomethasone (Clenil Modulite, Chiesi, Manchester, UK) were halved, and fluticasone doses of $250 \mu \mathrm{g}$ and $500 \mu \mathrm{g}$ were treated as equivalent to extrafine-particle beclomethasone 200 and $400 \mu \mathrm{g} ;{ }^{f}$ : SABA daily dose was defined as total prescribed SABA during baseline year divided by 365 . Reproduced from [19] with permission from the publisher. 
baseline variables, and statistical analyses were carried out via primary and secondary effectiveness outcomes. The analysis of baseline data revealed clinical and statistically significant differences between cohorts. A matched cohort analysis was therefore carried out using demographic and clinical matching criteria, as outlined in table 1. To maximise cohort sizes, a 1:2 matching was carried out between the extrafine-particle ICS step-up and the separate ICS+LABA cohorts on the one hand, and between the FDC fine-particle ICS/ LABA and the separate ICS+LABA cohorts on the other hand. Summary statistics were used to describe all matched baseline and outcome variables, both for the combined patient group and separated into treatment groups. To reduce the possibility of selection bias, numerous potential confounding variables were assessed, including some that were identified during previous respiratory research from the research team: baseline differences between treatment cohorts $(p<0.10)$ were considered potentially important, as well as baseline variables that were predictive $(\mathrm{p}<0.05)$ of each effectiveness outcome in multivariable analyses. Variables that met these criteria were examined for collinearity, as well as for clinical importance, to select those used as potential confounders in regression modelling of outcomes.

Two-way comparisons between cohorts, both baseline and outcome variables, were analysed by conditional logistic regression. The adjusted odds of achieving risk-domain asthma control were compared between treatment groups using conditional logistic regression models, with asthma control used as the dependent variable, and treatment and potential confounding factors as explanatory variables. Variables with $\mathrm{p}<0.10$ were included as potential confounding factors. The same method was employed to determine the adjusted odds of achieving treatment stability. The total number of severe exacerbations during the outcome period was compared between matched treatment groups using a conditional Poisson regression model, and exacerbation rate ratios were determined. The model used empirical standard errors for more robust confidence intervals, and adjustments were made for potential baseline confounders. Statistically significant results were defined as $\mathrm{p}<0.05$. Analyses were carried out using SPSS Statistics (version 19; IBM, Armonk, NY, USA), SAS (version 9.3; SAS Institute, Cary, NC, USA), and Microsoft Office Excel 2007 (Microsoft Corp. Redmond, WA, USA).

\section{Results}

Extrafine-particle ICS dose step-up compared with ICS and LABA in separate inhalers

Information on the unmatched patient population is available in the supplementary material and table S1. After patient selection (figure S1) and 1:2 matching there were 3232 patients in the extrafine ICS step-up cohort and 6464 patients in the separate ICS+LABA cohort (table 2, table S2, supplementary material). The mean age was 42 years and $61 \%$ were female. The cohorts were well matched for asthma-related parameters, and statistically significant differences between cohorts were small (table 2). The mean ICS dose prescribed on the index date for the extrafine ICS step-up cohort was approximately twice that for the separate ICS+LABA cohort (394 versus $194 \mu \mathrm{g} \cdot \mathrm{day}^{-1}$; $\mathrm{p}<0.001$ ).

Over the outcome year, the proportion of patients who achieved risk-domain asthma control increased from $65 \%$ during the baseline year to $75 \%$ and $71 \%$ in the extrafine ICS step-up and separate ICS+LABA cohorts, respectively. Therefore, a significantly higher proportion of patients prescribed extrafine ICS step-up achieved risk-domain asthma control compared with those prescribed separate ICS+LABA, and fewer of these patients experienced severe asthma exacerbations or acute respiratory events or required a change in therapy (table 3). The adjusted odds ratios for achieving risk-domain asthma control and treatment stability were significantly greater with extrafine ICS step-up compared with separate ICS+LABA. For risk-domain asthma control, the OR (95\% CI) was 1.25 (1.13-1.38), while the rates of severe exacerbations or acute respiratory events were significantly lower (figure 1).

The daily dose of SABA during the outcome year was significantly higher in the extrafine ICS step-up cohort than in the separate ICS+LABA cohort, although the median was the same $\left(219 \mu \mathrm{g} \cdot \mathrm{day}^{-1}\right)$. However, total $\beta_{2}$-agonist coverage was significantly longer for the separate ICS+LABA cohort than for the extrafine ICS step-up cohort (table 3, figure 2a).

Fine-particle ICS/LABA combination inhaler compared with ICS and LABA in separate inhalers After matching, there were 7529 patients in the fine-particle ICS/LABA combination cohort and 15058 patients in the separate ICS+LABA cohort. Most of the baseline differences between cohorts were small, although several were statistically significant because of the large cohort sizes (table 2, table S2, supplementary material). For the patients who received separate ICS+LABA inhalers, the mean index date was 2 years earlier than for those who received fine-particle ICS/LABA FDC inhalers. Probably as a consequence of this, fewer patients in the separate ICS+LABA cohort had recorded smoking data $(83 \%$ versus $92 \%$ in the ICS/LABA FDC cohort) (table 2).

During the outcome year, the proportion of patients who achieved risk-domain asthma control increased from $58 \%$ during the baseline year to $72 \%$ in the fine-particle ICS/LABA cohort and $68 \%$ in the separate 
TABLE 2 Baseline demographic and clinical characteristics for two two-way comparisons of a step-up in asthma therapy using an increased dose of extrafine-particle ICS or a fine-particle ICS/LABA combination inhaler, compared with add-on LABA in a separate inhaler

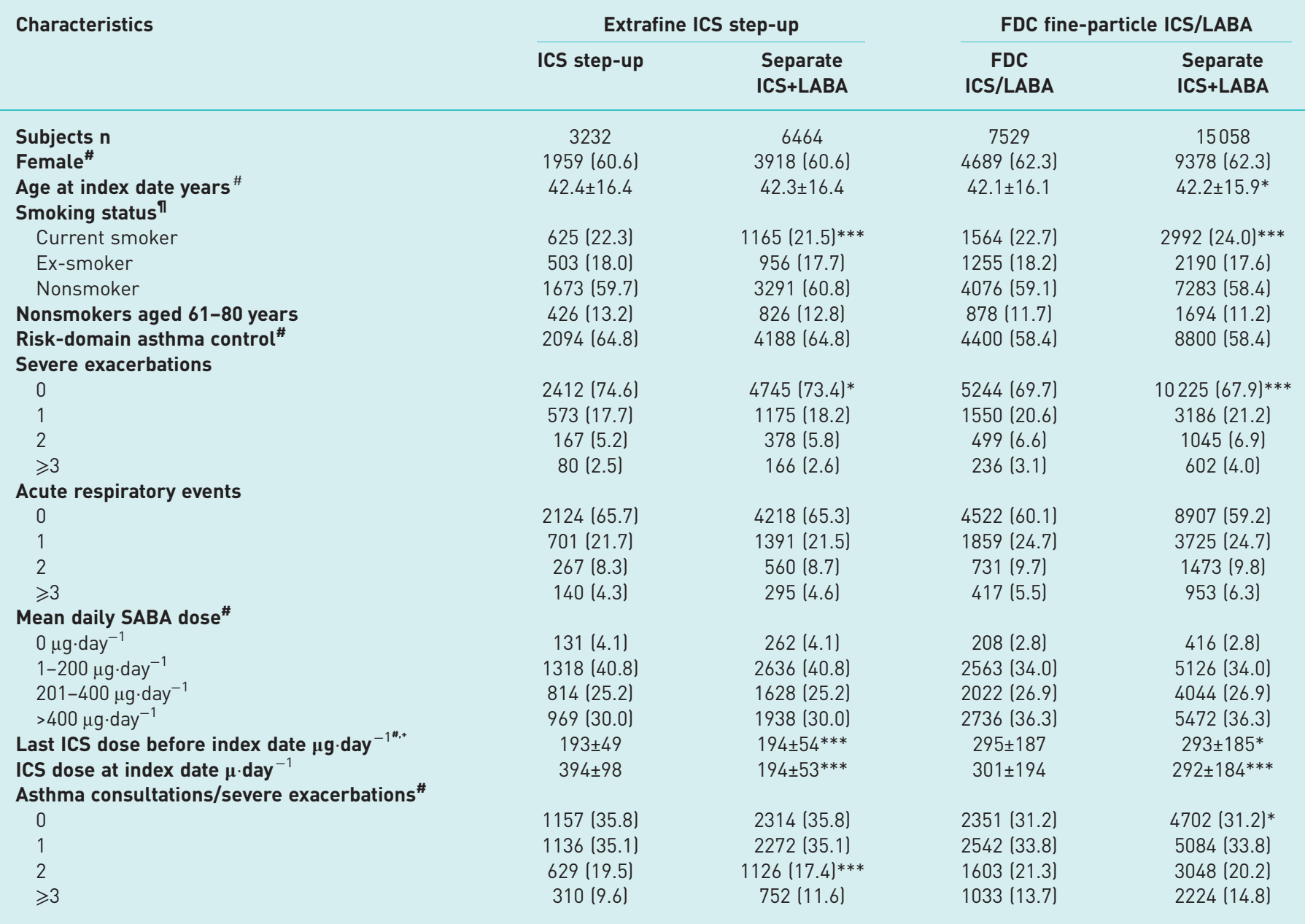

Data are presented as $\mathrm{n}(\%)$ or mean \pm SD, unless otherwise stated. Additional baseline data can be found in table S2. ICS: inhaled corticosteroids; LABA: long-acting $\beta_{2}$-agonist; FDC: fixed-dose combination; SABA: short-acting $\beta_{2}$-agonist.": matching variable; " : data were available for $2801(86.7 \%)$ and $5412(83.7 \%)$ patients in the ICS and separate ICS+LABA cohorts, respectively, and for 6895 (91.6\%) and 12465 $(82.8 \%)$ in the ICS/LABA combination and separate ICS+LABA cohorts, respectively; ${ }^{+}$: for the purposes of matching, doses of budesonide and large-particle beclomethasone (Clenil Modulite, Chiesi) were halved, and 250 and $500 \mu \mathrm{g}$ of fluticasone were set to be equivalent to 200 and $400 \mu \mathrm{g}$ of extrafine beclomethasone, respectively. At the index date, ICS doses are reported as half budesonide doses, while extrafine beclomethasone and fluticasone doses are reported without modification. ${ }^{*}: p \leqslant 0.05,{ }^{* * *} p<0.001$, conditional logistic regression for two-way comparison between cohorts.

ICS+LABA cohort. Risk-domain asthma control and treatment stability were significantly higher for patients who received fine-particle ICS/LABA inhalers compared with separate ICS+LABA inhalers. For risk-domain asthma control, the OR (95\% CI) was 1.06 (1.05-1.09) and the risk of severe exacerbations and acute respiratory events was significantly lower (figure 3, table 3). The proportion of patients changing treatment was also lower in the fine-particle ICS/LABA FDC cohort than in the separate ICS+LABA cohort (table 3).

The daily dose of SABA was significantly lower, and the daily $\beta_{2}$-agonist coverage (SABA+LABA) was significantly higher, for the fine-particle ICS/LABA FDC cohort compared with the separate ICS+LABA cohort (table 3 , figure $2 \mathrm{~b}$ ).

\section{Discussion}

This study of a real-life patient population showed that the odds of achieving risk-domain asthma control and treatment stability were significantly higher with an increased dose of extrafine-particle ICS or with fine-particle ICS/LABA FDC inhalers, as compared with ICS and LABA via separate inhalers. 
TABLE 3 Results from the 1-year outcome period from matched cohorts comparing a step-up in asthma therapy, using either an increased dose of extrafine-particle ICS, or a fine-particle ICS/LABA combination inhaler versus the addition of LABA to ICS in a separate inhaler

\begin{tabular}{|c|c|c|c|c|c|c|}
\hline \multirow[t]{2}{*}{ Outcome } & \multicolumn{2}{|c|}{ Extrafine ICS step-up } & \multirow[t]{2}{*}{ p-value ${ }^{f}$} & \multicolumn{2}{|c|}{ FDC fine-particle ICS } & \multirow[t]{2}{*}{ p-value ${ }^{f}$} \\
\hline & ICS step-up & Separate ICS+LABA & & FDC ICS/LABA & Separate ICS+LABA & \\
\hline Subjects $\mathbf{n}$ & 3232 & 6464 & & 7529 & 15058 & \\
\hline 0 & 2692 (83.3) & 5101 (78.9) & $<0.001$ & $6110(81.2)$ & $11441(76.0)$ & $<0.001$ \\
\hline 1 & $393(12.2)$ & 925 (14.3) & & 971 (12.9) & $2313(15.4)$ & \\
\hline \multicolumn{7}{|l|}{ Acute respiratory events } \\
\hline 0 & 2464 (76.2) & 4656 (72.0) & $<0.001$ & 5495 (73.0) & $10350(68.7)$ & $<0.001$ \\
\hline 1 & $528(16.3)$ & 1196 (18.5) & & 1343 (17.8) & 2939 (19.5) & \\
\hline 2 & 143 (4.4) & 355 (5.5) & & 404 (5.4) & 976 (6.5) & \\
\hline$\geqslant 3$ & $97(3.0)$ & $257(4.0)$ & & 287 (3.8) & 793 (5.3) & \\
\hline Treatment stability & $2111(65.3)$ & 3487 (53.9) & $<0.001$ & $4626(61.4)$ & $7862(52.2)$ & $<0.001$ \\
\hline$\geqslant 1$ asthma-related hospital attendance & $10(0.3)$ & $50(0.8)$ & 0.008 & $29(0.4)$ & $82(0.5)$ & 0.11 \\
\hline Additional therapy & $581(18.0)$ & $1385(21.4)$ & $<0.001$ & 469 (6.2) & $3344(22.2)$ & $<0.001$ \\
\hline ICS dose exposure $\mu \mathrm{g} \cdot$ day $^{-1 \Uparrow}$ & $274(164-438)$ & $155(65-247)$ & $<0.001$ & $197(115-345)$ & $192(96-329)$ & 0.008 \\
\hline Daily SABA dose $\mu \mathrm{g} \cdot$ day $^{-1}$ & $219(110-548)$ & $219(110-438)$ & $<0.001$ & $219(110-438)$ & $274(110-548)$ & $<0.001$ \\
\hline Daily $\beta_{2}$-agonist coverage $\mathrm{h}^{+}$ & $3.3(1.1-7.7)$ & $10.4(5.1-19.1)$ & $<0.001$ & $19.3(10.5-28.7)$ & $11.8(5.9-20.3)$ & $<0.001$ \\
\hline Change from baseline $h$ & $0.6(-0.5-2.3)$ & $7.0(2.9-14.7)$ & $<0.001$ & $15.2(7.4-23.7)$ & $7.6(2.9-15.2)$ & $<0.001$ \\
\hline Spacer device prescribed & $486(15.0)$ & 1182 (18.3) & $<0.001$ & $985(13.1)$ & $2926(19.4)$ & $<0.001$ \\
\hline Oropharyngeal candidiasis ${ }^{\S}$ & $155(4.8)$ & $321(5.0)$ & 0.72 & $396(5.3)$ & 819 (5.4) & 0.57 \\
\hline \multicolumn{7}{|c|}{$\begin{array}{l}\text { Data are presented as } \mathrm{n}(\mathrm{\%}) \text { or median (interquartile range), unless otherwise stated. ICS: inhaled corticosteroids; LABA: long-acting } \\
\beta_{2} \text {-agonist; FDC: fixed-dose combination; LRTI: lower respiratory tract infection; SABA: short-acting } \beta_{2} \text {-agonist. " : patients could have more } \\
\text { than one change in therapy land both increased ICS dose and additional therapyl during the year. Additional therapy could include combination } \\
\text { ICS/LABA inhaler (for ICS step-up cohort), separate LABA inhaler, leukotriene receptor antagonist and theophylline; }{ }^{\uparrow} \text { : the dose of budesonide } \\
\text { was halved for equivalence with extrafine beclomethasone and fluticasone, for which actual doses were used in the analyses. Daily ICS dose } \\
\text { exposure was calculated as the dispensed amount divided by } 365 \text {. In the separate ICS+LABA cohort, } 9-10 \% \text { of patients were prescribed } \\
\text { extrafine beclomethasone during the baseline period and } 10-11 \% \text { during the outcome period; }{ }^{+}: \text {defined as the dispensed amount divided by } \\
365 \text { and defining two puffs of SABA as lasting } 4 \mathrm{~h} \text {, and two puffs of LABA via pressurised metered-dose inhaler or one puff of LABA via dry } \\
\text { powder inhaler as lasting } 12 \mathrm{~h} \text {; }{ }^{\S} \text { : diagnosis of or therapy for oropharyngeal candidiasis (thrush); : p-value for conditional logistic regression. }\end{array}$} \\
\hline
\end{tabular}

Furthermore, the rates of severe exacerbations and acute respiratory events were significantly lower with increased doses of extrafine-particle ICS or fine-particle ICS/LABA FDC inhalers than with separate ICS+LABA inhalers. The total $\beta_{2}$-agonist coverage (adding hours covered by SABA+LABA) was significantly longer for patients prescribed add-on LABA.

National and international guidelines recommend add-on LABA as the first step-up of asthma therapy. Until 2011 (the last year of our study period) the recommendation of British guidelines for add-on LABA was either via ICS/LABA FDC inhaler or ICS+LABA via separate inhalers [3, 33]. In agreement with this, add-on LABA was the most commonly prescribed step-up therapy in the current study. Of the 35356 patients in the unmatched cohorts who met the eligibility criteria, $84 \%$ were prescribed add-on LABA, of whom more than two-thirds received ICS and LABA in separate inhalers.

Asthma management guidelines are often predominantly based on randomised controlled trials that exclude a large proportion of patients. Although the data are not always clear, several reports have suggested that there is no difference in efficacy between add-on LABA in combination or in separate inhalers $[16,33,34]$. This could partly be due to generally high adherence in these studies, which may therefore not reflect the situation in the general patient population of asthma patients, where adherence tends to be relatively low $[15,35,36]$. Differences in adherence probably contribute to the discrepancy between the efficacy shown in controlled trials and the effectiveness observed in real-life studies [19, 37, 38]. Real-life studies have indeed suggested that FDC inhalers can make a substantial contribution to aid refill persistence [18], and that patients on these inhalers are less likely to require rescue medication than 


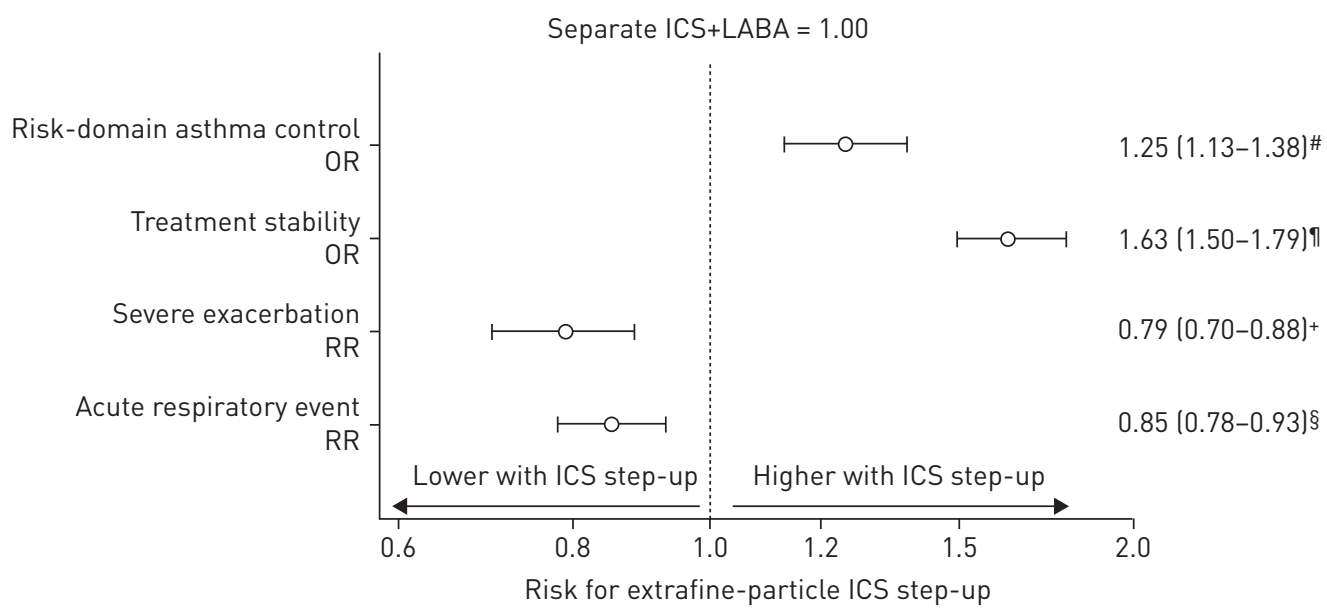

FIGURE 1 Adjusted outcome measures comparing a step-up in asthma therapy using an increased dose of extrafine-particle inhaled corticosteroids (ICS) versus the addition of a long-acting $\beta_{2}$-agonist (LABA) to ICS in a separate inhaler. Data show the adjusted odds ratio (OR) or rate ratio (RR) with $95 \% \mathrm{Cl}$. Variables have been adjusted for the following. " : number of oral corticosteroid prescriptions; ": smoking status and number of oral corticosteroid prescriptions; ${ }^{+}$: smoking status and number of severe exacerbations during baseline year; $\S$ : smoking status, number of oral corticosteroid prescriptions and number of primary care consultations.

those using separate inhalers [17]. Indeed, the current results show a substantial advantage of fine-particle ICS/LABA FDC inhalers compared with separate inhalers.

An alternative step-up option to add-on LABA is an increased dose of ICS, which has previously been associated with a reduction in serious adverse events and hospitalisation [39]. Our previous historical matched cohort study has shown that, in a general population of patients with asthma, an increased dose of extrafine-particle ICS is as effective as fine-particle ICS/LABA in a FDC inhaler [19]. Furthermore, the current study shows that an increased dose of extrafine ICS is significantly more effective than add-on LABA via separate inhalers. A potential concern of ICS step-up might be that this leads to increased use of reliever therapy. Although the statistical test shows that the daily dose of SABA was higher for patients prescribed increased extrafine ICS compared with those prescribed separate ICS+LABA, the median daily SABA dose was the same in both cohorts, suggesting that there is no real clinical difference between the groups. In contrast, the median daily SABA dose was substantially higher for patients prescribed separate ICS+LABA than for those prescribed fine-particle ICS/LABA in FDC inhalers (274 and $219 \mu \mathrm{g} \cdot \mathrm{day}^{-1}$, respectively). Furthermore, for total $\beta_{2}$-agonist coverage (SABA+LABA), the exposure was significantly longer for patients prescribed add-on LABA (median $3 \mathrm{~h}$ in the ICS step-up versus 10-19 $\mathrm{h}$ in the add-on
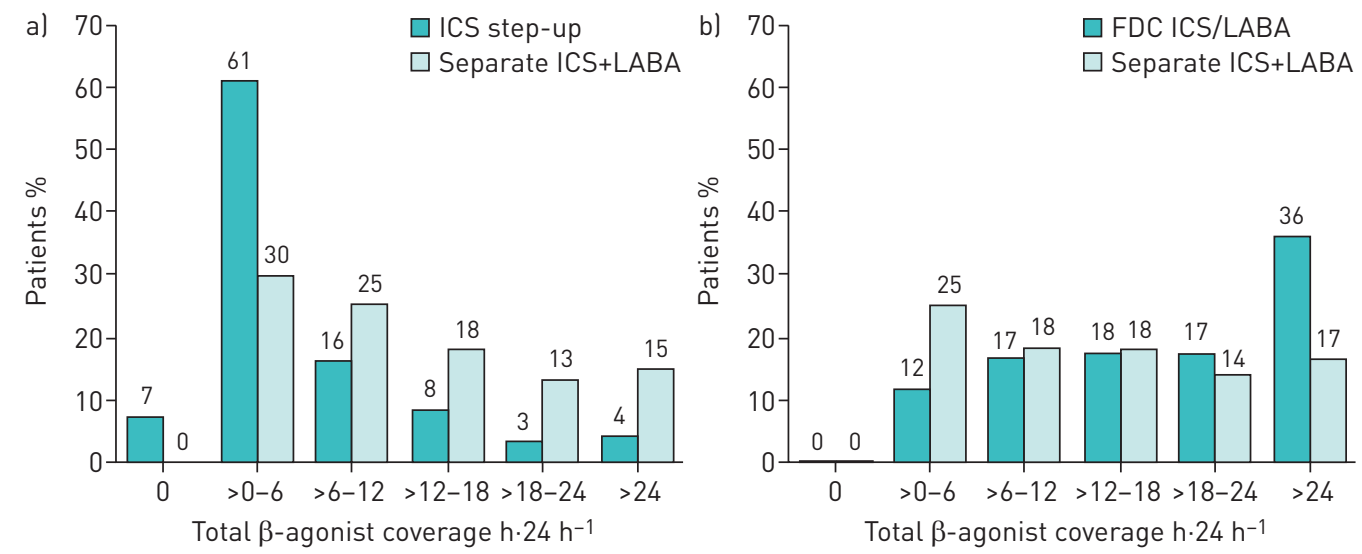

FIGURE 2 Comparison of total $\beta_{2}$-agonist coverage for patients prescribed al an increased dose of extrafine inhaled corticosteroids (ICS) versus ICS+long-acting $\beta_{2}$-agonist (LABA) in separate inhalers or b) fixed-dose combination (FDC) of fine-particle ICS/LABA inhalers versus ICS+LABA in separate inhalers. Data are presented as coverage in hours per $24 \mathrm{~h}$, where the total daily coverage of $\beta_{2}$-agonist (short-acting $\beta_{2}$-agonist (SABA)+LABA) was calculated as the annual dispensed amount divided by 365 and two puffs of SABA lalbuterol $200 \mu \mathrm{g}$ or terbutaline $500 \mu \mathrm{g}$ ) were defined as lasting $4 \mathrm{~h}$, and two puffs of LABA via pressurised metered-dose inhaler or one puff of LABA via dry powder inhaler were defined as lasting $12 \mathrm{~h}$. 


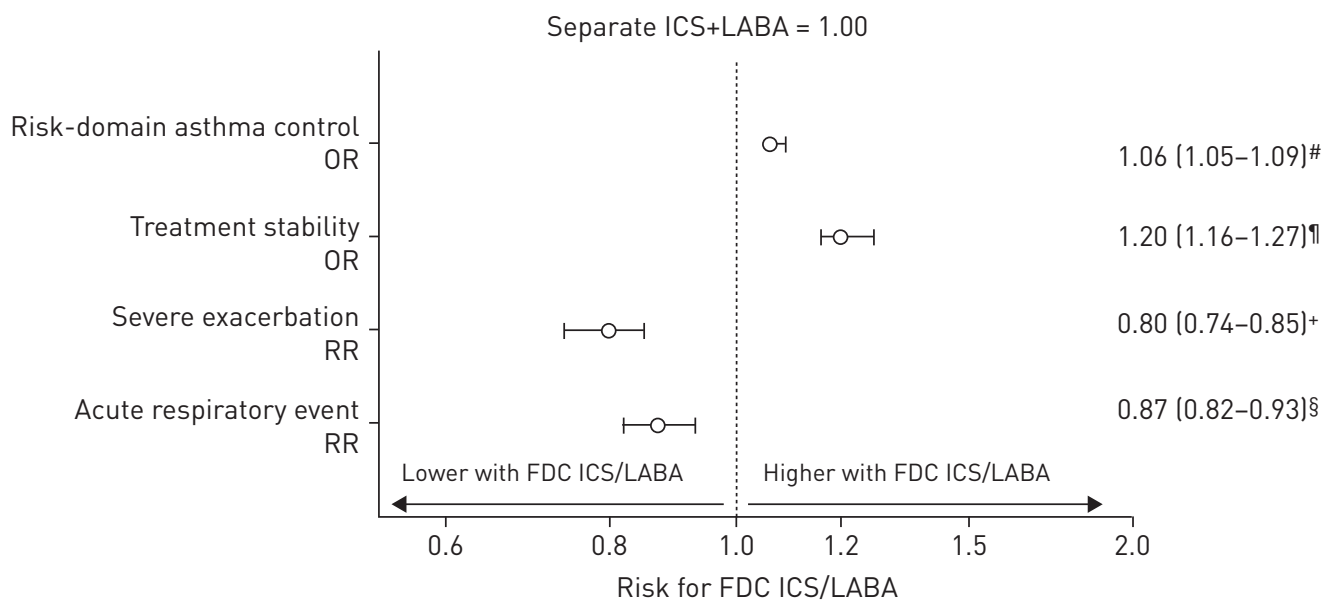

FIGURE 3 Adjusted outcome measures comparing a step-up in asthma therapy using a fixed-dose combination (FDC) inhaler containing fine-particle inhaled corticosteroid (ICS)/long-acting $\beta_{2}$-agonist (LABA) versus the addition of LABA to ICS in a separate inhaler. Data show the adjusted odds ratio (OR) or rate ratio (RR) with $95 \% \mathrm{Cl}$. Variables have been adjusted for the following. " : smoking status, number of primary care consultations and medication possession ratio; ": smoking status, adherence to ICS therapy, number of nonasthma-related consultations and number of acute respiratory events; ${ }^{+}$: number of acute respiratory events (categorised) and year of index date; ${ }^{\S}$ : number of acute respiratory events (categorised), year of index date and number of primary care consultations.

LABA cohorts). Therefore, an ICS dose step-up does not appear to result in any substantial increase in SABA prescription, while total $\beta_{2}$-agonist exposure increases substantially with add-on LABA.

In randomised controlled trials of bronchodilators, the primary end-point tends to be lung function, and patients generally have to demonstrate $\beta_{2}$-agonist reversibility to be included. The trials are also often limited in timescale, and most require patients with stable asthma whose exacerbation risk is low. In this context, the current study has several strengths. The findings depict clinically relevant real-life outcomes for asthma therapy in UK primary care, the patient group is larger than most controlled trials, and the 1 -year follow-up is likely to minimise seasonal variation and capture less frequent outcomes, such as exacerbations. We did not exclude active smokers (15-24\% of each cohort, except in the 61-80-year-old age group), patients did not have to demonstrate $\beta_{2}$-agonist reversibility to be included, and $\sim 25 \%$ of patients in each cohort had rhinitis, which is a risk factor for poor asthma control [40]. Therefore, the analysed patient group appears to be representative of the general UK asthma population, and this improves generalisability of the study findings to general practice.

Our primary end-point of risk-domain asthma control was designed to capture the absence of asthma exacerbations during the outcome year. This composite data outcome has been used in previous research $[19,24]$ and is in line with factors identified by expert working groups as being reflective of the future risk component of asthma control [31,32,41]. Although we conducted a matched cohort analysis, and confounding was eliminated to the greatest possible extent with adjusted analyses, we acknowledge that the use of matching may increase the risk of selection bias [42], and ignoring the matching variables may produce a certain population causal effect [43]. Similar to other historical cohort studies, this one is also limited by the fact that causal relationships cannot be inferred from the observed associations, and the possibility of unrecognised confounding factors remains. Nevertheless, we believe that, although slight baseline differences might still be present, they are unlikely to be of clinical significance.

For patients receiving ICS and LABA in separate inhalers, poor adherence to the ICS component can be associated with risks. An investigation into asthma deaths in the UK found that $6 \%$ of patients prescribed LABA in separate inhalers were not prescribed any ICS throughout the year, and an additional $12 \%$ had significant gaps in ICS prescribing, suggesting that approximately one in five of these patients were exposed to the increased risks associated with LABA monotherapy $[3,4]$. In clinical practice, it is generally considered that combination inhalers improve adherence, and they guarantee that LABA is not taken without ICS [2]. Our results provide real-life evidence to support the notion that either ICS step-up or add-on LABA via FDC inhalers is more effective than ICS and LABA in separate inhalers, which is the least desirable step-up option in a general patient population. The importance of this information is emphasised by the fact that more than two-thirds of patients prescribed add-on LABA received it via separate inhalers. 
In conclusion, this real-life study of a broad general population of young people and adults with persistent asthma suggests that step-up therapy, by increasing the dose of extrafine-particle ICS or by adding LABA in FDC inhalers, is a better approach than prescribing ICS and LABA in separate inhalers. Whether this is a strategy that will work better in some patient subgroups, e.g. patients with impaired therapeutic response to $\beta_{2}$-agonists or those with frequent exacerbations, is a question for further study.

\section{Acknowledgements}

We thank Neil Barnes (London Chest Hospital, London, UK) for his advice during the study design development and Anne Burden (Research in Real-Life Ltd, Cambridge, UK) for contributions to the study design and initial analyses. Our thanks go also to Alison Chisholm (Respiratory Effectiveness Group, Cambridge, UK) and Muzammil Ali (Research in Real-Life Ltd) for their assistance and input on data analyses and interpretation, and to Helga Mikkelsen (Cambridge Research Support, Cambridge, UK) and James Haslam (freelance) for writing and editorial assistance, funded by Research in Real-Life Ltd.

\section{References}

1 Global Initiative for Asthma. Global Strategy for Asthma Management and Prevention. 2015. Available from www.ginasthma.org

2 British Thoracic Society, Scottish Intercollegiate Guideline Network. British guideline on the management of asthma. A national clinical guideline. 2014. www.sign.ac.uk/guidelines/fulltext/141/ Date last accessed: May 18, 2016.

3 Morales DR. LABA monotherapy in asthma: an avoidable problem. Br J Gen Pract 2013; 63: 627-628.

4 Royal College of Physicians. Why asthma still kills: the National Review of Asthma Deaths (NRAD) Confidential Enquiry Report. London, RCP, 2014.

5 Lemanske RF Jr, Mauger DT, Sorkness CA, et al. Step-up therapy for children with uncontrolled asthma receiving inhaled corticosteroids. N Engl J Med 2010; 362: 975-985.

6 Ducharme FM, Ni Chroinin M, Greenstone I, et al. Addition of long-acting $\beta_{2}$-agonists to inhaled steroids versus higher dose inhaled steroids in adults and children with persistent asthma. Cochrane Database Syst Rev 4: 2010; CD005533.

7 Bisgaard $\mathrm{H}$. Effect of long-acting $\beta_{2}$-agonists on exacerbation rates of asthma in children. Pediatr Pulmonol 2003; 36: 391-398.

8 Greening AP, Ind PW, Northfield M, et al. Added salmeterol versus higher-dose corticosteroid in asthma patients with symptoms on existing inhaled corticosteroid. Allen \& Hanburys Limited UK Study Group. Lancet 1994; 344: 219-224.

9 Shrewsbury S, Pyke S, Britton M. Meta-analysis of increased dose of inhaled steroid or addition of salmeterol in symptomatic asthma (MIASMA). BMJ 2000; 320: 1368-1373.

10 Bateman ED, Boushey HA, Bousquet J, et al. Can guideline-defined asthma control be achieved? The Gaining Optimal Asthma ControL study. Am J Respir Crit Care Med 2004; 170: 836-844.

11 Pauwels RA, Lofdahl CG, Postma DS, et al. Effect of inhaled formoterol and budesonide on exacerbations of asthma. Formoterol and Corticosteroids Establishing Therapy (FACET) International Study Group. $N$ Engl J Med 1997; 337: 1405-1411.

12 Shepherd J, Rogers G, Anderson R, et al. Systematic review and economic analysis of the comparative effectiveness of different inhaled corticosteroids and their usage with long-acting $\beta_{2}$-agonists for the treatment of chronic asthma in adults and children aged 12 years and over. Health Technol Assess 2008; 12: 1-360.

13 O'Byrne PM, Naya IP, Kallen A, et al. Increasing doses of inhaled corticosteroids compared to adding long-acting inhaled $\beta_{2}$-agonists in achieving asthma control. Chest 2008; 134: 1192-1199.

14 Herland $\mathrm{K}$, Akselsen JP, Skjonsberg $\mathrm{OH}$, et al. How representative are clinical study patients with asthma or COPD for a larger "real life" population of patients with obstructive lung disease? Respir Med 2005; 99: 11-19.

15 Wu AC, Butler MG, Li L, et al. Primary adherence to controller medications for asthma is poor. Ann Am Thorac Soc 2015; 12: 161-166.

16 Perrin $\mathrm{K}$, Williams M, Wijesinghe $\mathrm{M}$, et al. Randomized controlled trial of adherence with single or combination inhaled corticosteroid/long-acting $\beta$-agonist inhaler therapy in asthma. J Allergy Clin Immunol 2010; 126: 505-510.

17 Kardos P. Budesonide/formoterol maintenance and reliever therapy versus free-combination therapy for asthma: a real-life study. Pneumologie 2013; 67: 463-470.

18 Stoloff SW, Stempel DA, Meyer J, et al. Improved refill persistence with fluticasone propionate and salmeterol in a single inhaler compared with other controller therapies. J Allergy Clin Immunol 2004; 113: 245-251.

19 Israel E, Roche N, Martin RJ, et al. Increased dose of inhaled corticosteroid versus add-on long-acting $\beta$-agonist for step-up therapy in asthma. Ann Am Thorac Soc 2015; 12: 798-806.

20 Leach CL, Kuehl PJ, Chand R, et al. Respiratory tract deposition of HFA-beclomethasone and HFA-fluticasone in asthmatic patients. J Aerosol Med Pulm Drug Deliv 2016; 29: 127-133.

21 Leach CL, Kuehl PJ, Chand R, et al. Characterization of respiratory deposition of fluticasone-salmeterol hydrofluoroalkane-134a and hydrofluoroalkane-134a beclomethasone in asthmatic patients. Ann Allergy Asthma Immunol 2012; 108: 195-200.

22 Barnes N, Price D, Colice G, et al. Asthma control with extrafine-particle hydrofluoroalkane-beclometasone vs. large-particle chlorofluorocarbon-beclometasone: a real-world observational study. Clin Exp Allergy 2011; 41: $1521-1532$

23 Price D, Thomas M, Haughney J, et al. Real-life comparison of beclometasone dipropionate as an extrafine- or larger-particle formulation for asthma. Respir Med 2013; 107: 987-1000.

24 Colice G, Martin RJ, Israel E, et al. Asthma outcomes and costs of therapy with extrafine beclomethasone and fluticasone. J Allergy Clin Immunol 2013; 132: 45-54.

25 Price D, Martin RJ, Barnes N, et al. Prescribing practices and asthma control with hydrofluoroalkane-beclomethasone and fluticasone: a real-world observational study. J Allergy Clin Immunol 2010; 126: 511-518. 

876-884.

35 Marceau C, Lemiere C, Berbiche D, et al. Persistence, adherence, and effectiveness of combination therapy among adult patients with asthma. J Allergy Clin Immunol 2006; 118: 574-581.

36 Holgate ST, Price D, Valovirta E. Asthma out of control? A structured review of recent patient surveys. BMC Pulm Med 2006; 6: Suppl. 1, S2.

37 Price D, Musgrave S, Wilson E, et al. A pragmatic single-blind randomised controlled trial and economic evaluation of the use of leukotriene receptor antagonists in primary care at steps 2 and 3 of the national asthma guidelines (ELEVATE study). Health Technol Assess 2011; 15: 1-132.

Price D, Chisholm A, van der Molen T, et al. Reassessing the evidence hierarchy in asthma: evaluating comparative effectiveness. Curr Allergy Asthma Rep 2011; 11: 526-538.

39 Thomas M, Price D, Chrystyn H, et al. Inhaled corticosteroids for asthma: impact of practice level device switching on asthma control. BMC Pulm Med 2009; 9: 1.

40 Greiner AN, Hellings PW, Rotiroti G, et al. Allergic rhinitis. Lancet 2011; 378: 2112-2122.

41 Fuhlbrigge A, Peden D, Apter AJ, et al. Asthma outcomes: exacerbations. J Allergy Clin Immunol 2012; 129: Suppl. 3, S34-S48.

42 Rose S, Laan MJ. Why match? Investigating matched case-control study designs with causal effect estimation. Int $J$ Biostat 2009; 5: Article 1.

43 Sjolander A, Greenland S. Ignoring the matching variables in cohort studies - when is it valid and why? Stat Med 2013; 32: 4696-4708. 